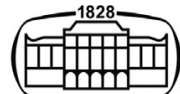

AKADÉMIAI KIADÓ

IMAGING

CASE REPORT

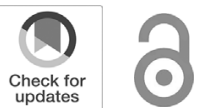

IMAGING 13 (2021) 1, 80-83

DOI: $10.1556 / 1647.2020 .00008$

(C) 2020 The Author(s)

* Corresponding author.

Tel.: $+390871 / 357999$

fax: $+390871 / 358982$.

E-mail: luca.procaccini93@gmail.com

\title{
Klippel-Trenaunay syndrome (KTS): A report of two patients and review of literature
}

\author{
LUCA PROCACCINI $^{1 *} \odot$, BRUNO CONSORTE ${ }^{1}$, \\ DANIELA GABRIELLI ${ }^{2}$, ANTONIETTA CIFARATTI ${ }^{1}$ and \\ MASSIMO CAULO ${ }^{1}$
}

\footnotetext{
${ }^{1}$ Department of Neuroscience and Imaging and Clinical Sciences, Institute of Radiology, Section of Diagnostic Imaging and Therapy-Radiology Division, "G. d'Annunzio" University, Chieti-Pescara, Vestini Street, Chieti, 66100, Italy

${ }^{2}$ Unit of Interventional Radiology, “C. e G. Mazzoni” Hospital, AV5 - ASUR Marche, Ascoli Piceno, Italy
}

Received: November 29, 2020 • Accepted: December 7, 2020

\begin{abstract}
Klippel-Trenaunay syndrome (KTS) is an uncommon congenital condition, resulting in vascular malformations affecting capillary, venous, and lymphatic systems and bone and/or soft tissue hypertrophy. Magnetic Resonance Angiography (MRA) may be useful in assessing the severity of the disease and for treatment planning. We present two cases of two white men with the typical clinical presentation of Klippel-Trenaunay syndrome i.e. vascular malformations (capillary, venous and lymphatic) and localized bone and/or soft tissues hypertrophy. Splenic hemangiomas were evidenced in both patients and MRA was helpful in assessing and delineating the abnormal venous drainage system. KTS is a complex disorder whose true prevalence and etiology are still unknown. In most cases the emblematic clinical manifestation consisting in vascular malformations and extremity overgrowth is represented. KTS may be associated with several different conditions including scoliosis and splenic hemangiomas. The presence of the lateral marginal vein (LMV) is pathognomonic. Imaging is fundamental in confirming the diagnosis and for therapeutic strategies. An effective treatment does not exist to date and a multidisciplinary approach is usually required to prevent complications.
\end{abstract}

\section{KEYWORDS}

Klippel-Trenaunay syndrome (KTS), Varicose veins, Magnetic Resonance Angiography (MRA), Lower limb hypertrophy, Port-wine stains, Lateral marginal vein (LMV)

\section{Introduction}

Klippel-Trenaunay syndrome (KTS) is characterized by the classic triad of capillary malformations manifesting as a "port-wine stain", venous varicosities and bone and/or soft tissue hypertrophy. Described in 1900 by Maurice Klippel and Paul Trenaunay [1], etiology, pathogenesis and genetics are poorly understood. Its incidence is about 1:100.000 with no ethnic or sex predilection and usually affects unilateral lower limb; however, various systemic organs may be involved.

Multidisciplinary approach is necessary. Diagnosis is a challenge due to the complexity and variability of manifestations and is made clinically when at least two of the three cardinal features are present [2]. Imaging techniques are mandatory to assess its severity and choice the treatment strategy [3].

\section{Case 1}

A 49-year-old male with a history of KTS diagnosed at the age of two, was admitted to our hospital with right lower limb pain. He showed "port-wine stain" and varicose veins 


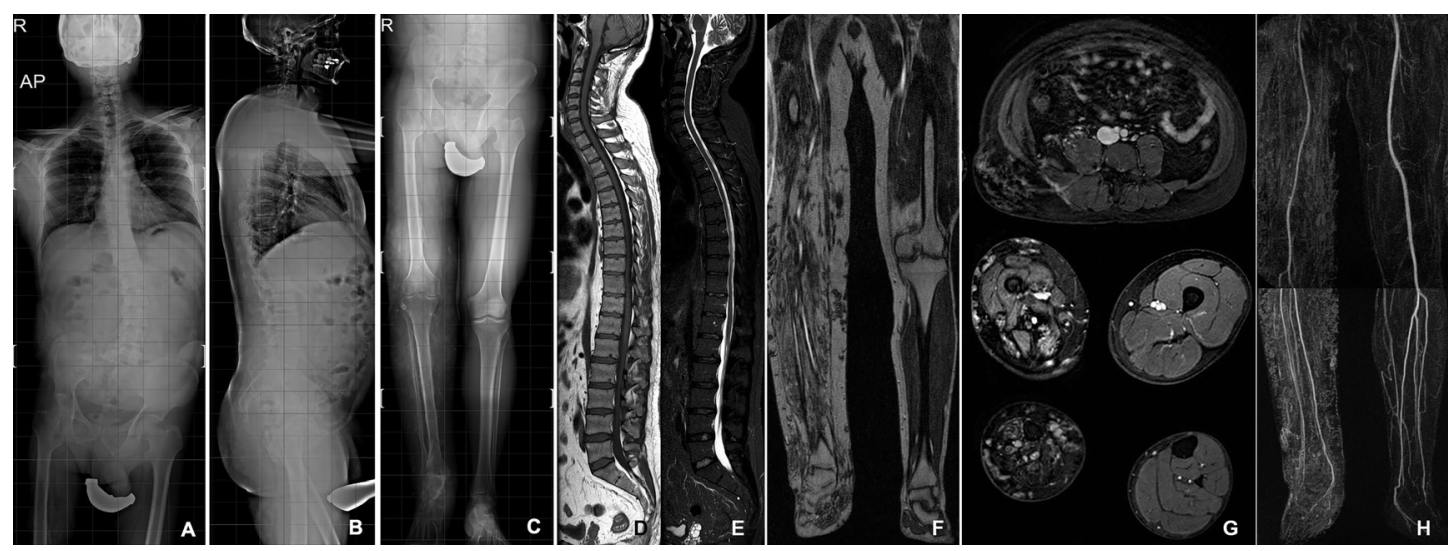

Fig. 1. (A) Standing anteroposterior and (B) lateral radiographs. (C) Radiography shows limb length discrepancy (LLD), right coxa valga and periosteal and endosteal scalloping of the right femur, tibia and fibula. Soft tissue hypertrophy of the right lower limb associated to multiple vascular calcifications are detected. (D, E) T1 and T2+FS without contrast in sagittal views: spinal disc herniation or Chiari-I malformation are not documented. (F) T1 and (G) T1+FS with contrast in coronal and axial views, respectively, well depict the right lower limb hypertrophy, the fatty replacement of all muscles, subcutaneous and intermuscular lesions from gluteal region to the foot representing diffuse soft tissue hemangiomas. (H) Contrast-enhanced MRA three-dimensional reconstructed maximum intensity projection shows normal arterial course bilaterally

associated to severe hypertrophy of the leg. Past surgical history revealed tibio-tarsal arthrodesis in 1993, and multiple vascular malformations excisions. Radiographies showed skeletal KTS manifestations (Fig. 1). MRI of the spine not documented Chiari-I malformation. The study was completed by Magnetic Resonance Angiography (MRA) of the lower extremity which demonstrated multiple, irregular, prematurely contrast-enhanced venous varicosities both in the superficial and deep venous system, from the gluteal region to the foot. A significant unilateral lower limb hypertrophy with fatty muscle degeneration was evidenced (Fig. 1). Conservative treatment was advised, consisting in elastic compression stockings, shoe lift and pain medications as needed.

\section{Case 2}

A 47-year-old male diagnosed with KTS in 1997 at the age of 24, was admitted to our department with right lower limb increasing heaviness, numbness and swelling. He showed multiple varicose veins, ulcerations, "port-wine stain" and enlargement of the extremity. Varicose eczema, phlebetic lymphedema referring to chronic venous insufficiency were also noticed. In 1999 a venography was performed which revealed the presence of the Lateral Marginal Vein (LMV) excised after ten years cause the worsening of symptoms. In 2018 the progression of such condition led to C4 score according to CEAP (Clinical-Etiological-Anatomical-Pathophysiological) classification which required a duplex ultrasonography (DUS); main findings were the incompetence of the right saphenopopliteal junction, numerous collateral varicose veins and ectasia of the small saphenous vein. Thereafter, in 2019, MRA demonstrated multiple venous varicosities in the middle third of the thigh and the leg as well as lower limb hypertrophy (Fig. 2). As a collateral finding, multiple hyperintense focal lesions on T2-weighted images were found in the spleen, referring to hemangiomas
(Fig. 2). Conservative treatment was undertaken, consisting of decongestive physiotherapy, manual lymphatic drainage and elastic compression stockings.

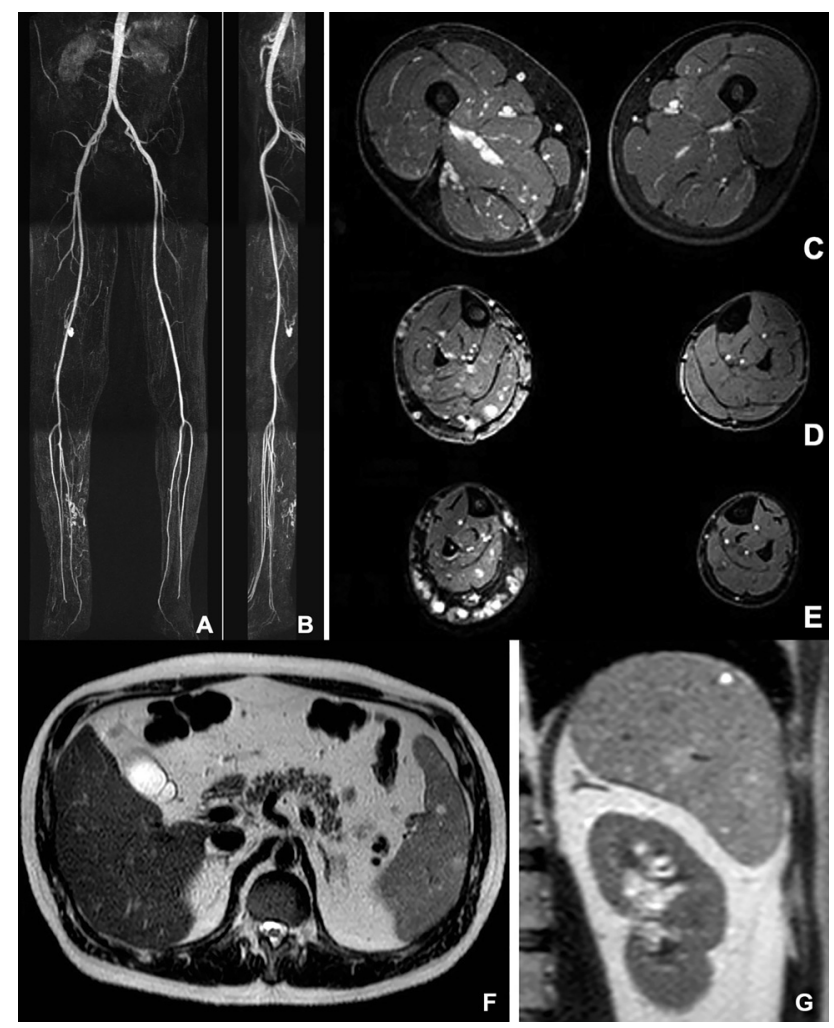

Fig. 2. (A, B) Contrast-enhanced MRA three-dimensional reconstructed maximum intensity projection in coronal and sagittal views and $(C, D, E) T 1+F S$ with contrast in axial views show right lower limb hypertrophy and multiple venous varicosities in the middle third of the thigh and the leg, localized posteriorly and postero-medially respectively. (F, G) T2 without contrast in axial and coronal views shows splenic hemangiomas, not splenomegaly. 


\section{Discussion}

KTS is a congenital disorder characterized by vascular malformations and localized bone and/or soft tissues overgrowth. Etiology is still unknown although sporadic chromosomal translocations have been reported; furthermore, KTS may be related to the PIK3CA-related overgrowth spectrum (PROS) [4].

Clinical manifestations are variable. Unilateral lower extremity is the common site involved, although any part of the body may be affected [5].

Venous malformations may relate both superficial and deep venous system.

Superficial varicosities usually involve the lateral side of the affected limb [6]; they may be associated with valvular incompetence, hypoplasia, aplasia, and deep venous aneurysms.

Persistent Embryonic Veins (PEVs) are characteristic for KTS: Lateral Marginal Vein (LMV, also called "vein of Servelle", "Klippel-Trenaunay vein") and Persistent Sciatic Vein (PSV), both valveless dilated veins that usually regress before birth. Their prevalence rate ranges from 9 to 68\% [7]. As for the deep venous system, KTS patients are more susceptible to recurrent deep vein thrombosis that may lead to acute and/or chronic thromboembolism.

Venous anomalies are commonly related to lymphatic malformations (LMs) with a prevalence rate ranging from 10 to $84 \%$. LMs may be macrocystic, microcystic and mixed and may lead to lymphatic insufficiency, congestion and lymphorrhea.

Various systemic organs may be involved including brain, cardiovascular system, gastrointestinal tract (more frequently distal colon and rectum) and genitourinary tract (anterior bladder wall, dome and external genitalia) [8].

Splenic hemangiomas in association with hypersplenism have also been reported [9]. It is worth remembering that scoliosis is frequently related with Chiari-I malformation with the prevalence rate from 15 to $50 \%$.

The diagnosis of KTS is clinical but imaging techniques may be helpful to confirm the diagnosis and to choose the treatment. Radiographies are used for skeletal manifestations (bony hypertrophy, LLD, scoliosis, polydactyly, syndactyly, and congenital hip dislocation). DUS is the first-level imaging technique for the evaluation of venous malformations and varicosities while CTA is useful to assess bone abnormalities and vascular malformations but it provides few information about muscle and surrounding soft tissue involvement. MRA is the imaging modality of choice for assessing the extent of vascular malformations, their relationship with adjacent structures and for pre-operative planning. It may depict multiple, prematurely contrastenhanced venous varicosities both in the superficial and deep venous system, muscle hypertrophy and/or fatty muscle degeneration and bone marrow changes.

Multidisciplinary approach is mandatory. Medical treatment is the first choice and includes local wound care, elevation and compression of the affected limb to prevent edema and ulcerations. The decongestive lymphatic therapy is a valid tool to avoid lymphedema complications. Anticoagulation should be administered if patients develop deep venous thrombosis and low-dose aspirin should be used if limb pain and swelling are refractory. Some selected patients have recently benefited from Sirolimus which inhibits the $\mathrm{PI} 3 \mathrm{~K} / \mathrm{mTOR}$ pathway [10].

LLD may be treated with shoe lift or compensatory shoes whether discrepancies are $<1.5 \mathrm{~cm}$, if $>2 \mathrm{~cm}$ osteotomy or epiphysiodesis may be required.

Vascular therapeutic interventions should be considered when the severity of KTS progresses to greater than CEAP class 3. The role of interventional radiology (embolization, sclerotherapy and radiofrequency ablation) is not well established but it is alternative to surgical treatment. Patients eligible for surgery are refractory to conservative care and/or manifest bleeding complications.

In conclusion, imaging should guide any therapeutic option to have a comprehensive overview of the situation.

Authors' contribution: L.P. B.C D.G. A.C. and M.C. contributed to the design, implementation and to the writing of the manuscript. All authors reviewed the final version of the manuscript and agreed to submit it to IMAGING for publications.

Funding sources: No financial support was received for this study.

Conflicts of interest: The authors have no conflict of interest to disclose.

Patient informed consent: Both patients gave written consent for publication of their identifiable details and images.

\section{ACKNOWLEDGMENTS}

None.

\section{REFERENCES}

[1] Gloviczki P, Driscoll DJ. Klippel-Trenaunay syndrome current management. Phlebology 2007; 22(6): 291-298.

[2] Tian XL, Kadaba R, You SA, Liu M, Timur AA, Yang L, et al.: Identification of an angiogenic factor that when mutated causes susceptibility to Klippel-Trenaunay syndrome. Nature 2004; 427(6975): 640-645.

[3] Sharma D, Lamba S, Pandita A, Shastri S: Klippel-Trénaunay syndrome-a very rare and interesting syndrome. Clin Med Insights Circ Respir Pulm Med 2015; 9: 1.

[4] Vahidnezhad H, Youssefian L, Uitto J: Klippel-Trenaunay syndrome belongs to the PIK3CA-related overgrowth spectrum (PROS). Exp Dermatol 2016; 25(1): 17-19. 
[5] Kihiczak GG, Meine JG, Schwartz RA, Janniger CK: KlippelTrenaunay syndrome: a multisystem disorder possibly resulting from a pathogenic gene for vascular and tissue overgrowth. Int J Dermatol 2006; 45(8) :883-890.

[6] Richter A, Wissgott C, Steinkamp HJ: Thrombophlebitis der vena embryonalis lateralis persistens. Rofo 2007; 179: 750-751.

[7] Rovira OJ, Repollet-Otero C, Rodriguez LE, Martinez-Trabal JL: Symptomatic, unilateral, isolated, complete persistent sciatic vein. J Vasc Surg Venous Lymphat Disord 2018; 6(1): 104-106.
[8] Abdel Razek AAK. Imaging findings of Klippel-Trenaunay syndrome. J Comput Assist Tomogr 2019; 43: 786-792.

[9] Pakter RL, Fishman EK, Nussbaum A, Giargiana FA, Zerhouni EA: CT findings in splenic hemangiomas in the Klippel- TrenaunayWeber syndrome. J Comput Assist Tomogr 1987; 11(1): 88-91.

[10] Hammer J, Seront E, Duez S, Dupont S, Van Damme A, Schmitz $S$, et al.: Sirolimus is efficacious in treatment for extensive and/or complex slow-flow vascular malformations: a monocentric prospective phase II study. Orphanet J Rare Dis 2018; 13(1): 191. 